\title{
An overview of COVID-19 infection in dental practices - a questionnaire survey
}

SADJ August 2021, Vol. 76 No. 7 p404 - p408

H Gluckman', CC Pontes ${ }^{2}$, M Troelzsch ${ }^{3}$, ET Scheyer ${ }^{4}$

\section{ABSTRACT}

\section{Introduction}

Dental nurses and practitioners are at high risk of exposure to COVID-19 due to physical proximity and exposure to body fluids during treatment. Dental practices have implemented multiple protective protocols to decrease COVID-19 transmission; however, it is difficult to evaluate how effective these measures are, as there is limited data on COVID-19 in dental practices.

\section{Aims and objectives}

To evaluate COVID-19 infection rates among dentists, dental staff, and patients in different countries through an online survey, with a primary focus on South Africa $(\mathrm{SA})$.

\section{Design}

Cross-sectional online survey.

Results

One hundred fifty-four participants from 52 countries answered the survey, $48.6 \%(n=561)$ from SA. COVID-19 infections were reported in $18.2 \%(n=210)$ of dental practices. Only $1.1 \%$ regarded the practice as the source of infection for dentists and staff who got infected.

\section{Author affiliations:}

1. Howard Gluckman: $P h D, B D S, M C h D$, Specialist in Periodontology, Implantology and Oral Medicine; Director, Implant and Aesthetics and The Implant Clinic, Cape Town, South Africa; Adjunct Assistant Professor at the School of Dental Medicine, Univ. of Pennsylvania, Pennsylvania, United States of America.

2. Carla C Pontes: $B D S, M S c, P h D$, Research Associate, The Implant Clinic, Cape Town, South Africa. ORCID Number: 0000-0002-9337-3783

3. Matthias Troelzsch: $D M D, M D$, Private practitioner at the Clinic for Oral and Maxillofacial Surgery, Ansbach, Germany. ORCID Number: 0000-0002-6027-2919

4. E Todd Scheyer: $D D S, M S$, Private practitioner in Periodontics and Implant dentistry, PerioHealth Professionals, Director for The McGuire institute for Clinical Research, Faculty at: iHDS, UTDB and guest Faculty at UTHSCSA, Houston, Texas.

Corresponding author: Howard Gluckman

The Implant Clinic, 39 Kloof Street, Gardens, 8001 Cape Town, South Africa.

Email: docg@mweb.co.za

Author contributions:

1. Howard Gluckman: Conceptualization, methodology, data curation $-25 \%$

2. Carla C Pontes: Methodology, writing - original draft, review and editing, formal analysis - $25 \%$

3. Matthias Troelzsch: Project administration, validation, resources $-25 \%$

4. E Todd Scheyer: Project administration, validation, resources $25 \%$
In total, $13.9 \%(n=160)$ treated COVID-19 patients. SA presented a higher infection rate (19\% vs $13 \%, p=0.04)$ and more frequent treatment of COVID-19 patients than the other countries combined ( $17 \%$ vs $11 \%, p=0.006)$.

\section{Conclusion}

These findings support the need to maintain strict infec tion control measures to decrease transmission of SARSCoV-2 during the delivery of oral care.

\section{INTRODUCTION}

As the coronavirus disease pandemic unfolds with the second wave of infections, dental professionals have been faced with numerous challenges to maintain oral care delivery. ${ }^{1}$ Because droplets composed of saliva and respiratory secretions constitute the leading route of transmission for SARS-CoV-2, the oral cavity plays a pivotal role in acquiring the virus and spread of the disease. ${ }^{2}$

While oral care is an essential part of healthcare, concerns have been raised about the transmission of the virus between dental practitioners, other dental staff members, and patients when undergoing dental procedures. ${ }^{3}$ According to the Office for National Statistics (ONS) from the UK, dental nurses and dental practitioners, are among the professionals who present the highest risk for exposure to COVID-19 due to the physical proximity during dental treatment and frequent exposure to body fluids. ${ }^{4}$

The World Health Organization (WHO) has recommended the postponement of elective dental procedures to mitigate disease transmission. Their interim guideline to only perform urgent or emergency dental procedures is based on cross-infection potential through aerosols. ${ }^{3}$ In many dental practices, there has been a decreased number of appointments, and reduced working hours, with patients experiencing an increased burden of oral disease, which is detrimental to general health. ${ }^{5}$

Dental practices around the globe have implemented multiple protective protocols to decrease COVID-19 transmission, according to guidelines from the $\mathrm{WHO},{ }^{3}$ the US Centre for Disease Control (CDC), ${ }^{6}$ the American Dental Association (ADA), ${ }^{7}$ and from national regulato- 
ry bodies in different countries. It is difficult to evaluate the effectiveness of the adopted infection control measures, as currently there is limited data on COVID-19 infections in dental practices. Among US dentists, the ADA has recently estimated the prevalence of COVID -19 infection to be below 1\%. In Brazil, the National Dentistry Council, reported that only $0.2 \%$ of COVID-19 infections in the country affected dental professionals. ${ }^{9}$

The present study aimed at evaluating COVID-19 infections among dentists, dental staff, and patients in different countries through an online survey, with the main focus on South Africa. This study's results can contribute to a better understanding of the risks for transmission of coronavirus disease related to oral healthcare delivery.

\section{MATERIAL AND METHODS}

\section{Study design}

This study was designed as an anonymous cross-sectional online survey developed by the South African Implant and Aesthetic academy using Survey Monkey ${ }^{\mathrm{TM}}$ (SVMK Inc. San Mateo, CA, US). The questionnaire was available from $15^{\text {th }}$ August 2020 to $15^{\text {th }}$ September 2020, being composed of nine questions.

\section{Questionnaire}

An electronic informed consent to voluntarily participate in the study was embedded in the first question. Questions 2 and 3 concerned workplace type and physical location. In question 4, the profession of the respondent was recorded. Questions 5 to 9 were related to COVID-19 infections in the participant's dental practice, treatment of COVID-19 patients, and patient infection rate after visiting the practice.

\section{Participant recruitment}

A South African dental academy alumni group (total of 445 members, anonymized as per submission request) was invited to participate in the survey via mobile instant messing and were encouraged to share the survey with their peers.

\section{Data analysis}

The answers to the survey were automatically recorded by the SurveyMonkeyTM software and exported as Excel spreadsheets. Data were presented as absolute and relative frequency. The data was analyzed in STATA 15.1 (StataCorp LLC, Texas, US) according to the physical location. The chi-square test was used to analyze differences in frequencies for South African participants compared to other countries' participants. An alpha value below 0.05 was considered statistically significant.

\section{RESULTS}

\section{Demographics}

In total, 1154 participants representing 52 different countries answered the online survey. With regards to the location of the participant's dental practice, nearly half were located in South Africa (48.6\%, $n=561)$, followed by United States (17.2\%, $n=199)$, and Belgium (7.9\%, $n=91$, Figure 1).

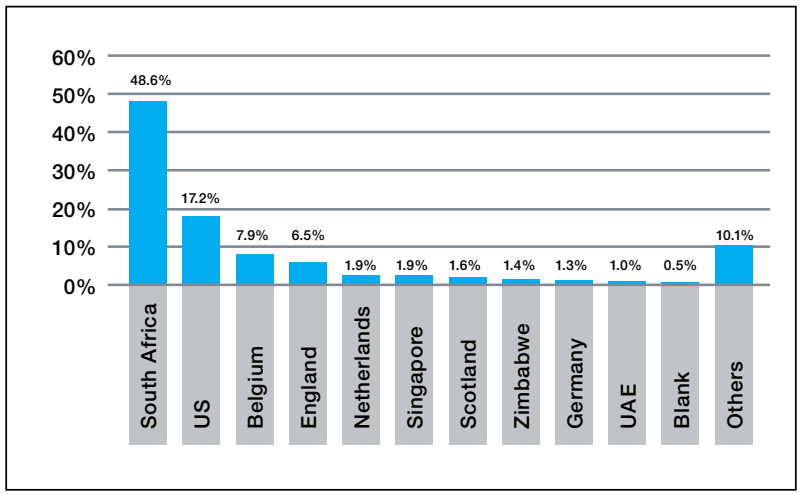

Figure 1. Survey participants according to country.

In total, $70.1 \%$ of the respondents were general dentists $(n=809), 24.6 \%$ were specialist dentists $(n=284), 2.2 \%$ were dental assistants or dental nurses, and the remaining 3.1\% $(n=36)$ represented other staff.

Regarding the workplace, the majority $(89.4 \%, n=1032)$ worked at private dental clinics, $6.9 \%(n=79)$ worked both at private and public dental clinics, $2.7 \%(n=31)$ worked at public dental clinics, and $1.0 \%(n=12)$ had other work settings.

\section{COVID-19 infection - dentists, dental} assistants, and dental staff

The majority of the participants reported no COVID-19 infections in their practices $(78.2 \%, n=902)$. In total, $18.2 \%$ $(n=210)$ reported COVID-19 infections in their practices, from which $88.5 \%(n=186)$ were confirmed by a laboratory test, and $11.5 \%(n=24)$ lacked diagnostic confirmation.

Less than $4 \%$ of the sample did not know if there were COVID-19 infections in their practices. When South Africa's responses were compared to the rest of the world (Figure 2), South Africa presented a higher infection rate than the other countries combined (19\% versus $13 \%, p=0.04$, Figure 2).

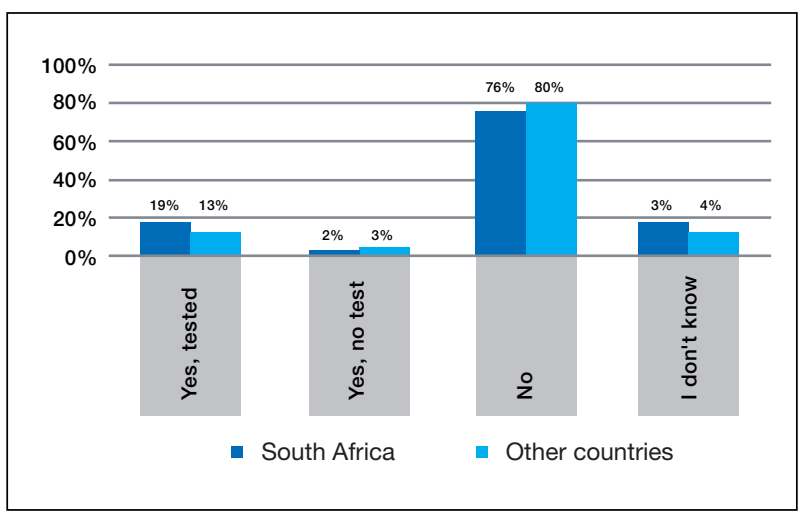

Figure 2. COVID-19 infections in respondent's practices according to the location $(p=0.04)$.

When asked about how the respondent or clinic staff acquired COVID-19 infection, 11.2\% $(n=137)$ answered 
'outside the practice', 10.3\% ( $\mathrm{n}=119)$ did not know, 1.1\% $(n=13)$ answered 'inside the practice', and $77.4 \%$ report no infections.

\section{COVID-19 infection - patients}

Regarding COVID-19 infection of patients after visiting dental practices, $91.8 \%$ of respondents had no reported patient infection, 3.8\% did not know, 3.6\% reported having patients infected 1-2 weeks after visiting the practice, as confirmed by laboratory tests, and $0.8 \%$ reported unconfirmed patient infection.

According to the survey, $13.9 \%(n=160)$ of the participants reported treatment of laboratory-confirmed COVID -19 patients. When evaluated by location, $17 \%$ of South African respondents treated laboratory-confirmed COVID -19 patients compared to $11 \%$ of respondents from other countries $(p=0.006)$.

There were no statistically significant differences in infection rates for respondents who reported treatment of confirmed cases in their practices $(14 \%, n=22$ infections reported by 160 participants) and those who did not treat confirmed COVID-19 patients (19\%, n=188 infections reported by 994 participants, $p=0.127)$.

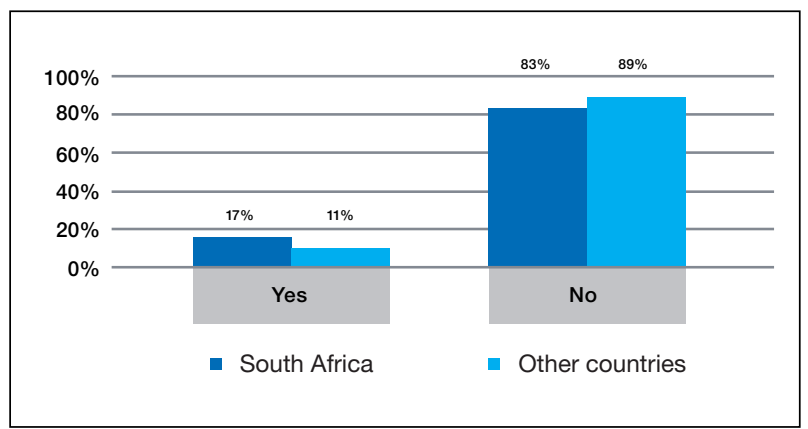

Figure 3. Treatment of laboratory-confirmed COVID-19 positive patients at respondent's practices according to the location $(p=0.006)$.

\section{DISCUSSION}

The majority of the participants were dental practitioners who work in private practices, with nearly half being located in South Africa. In total, $18.2 \%$ of the participants reported COVID-19 infection in their practices, and the majority of infections were confirmed by a laboratory test (88.5\%). Only $1.1 \%$ of the participants reported infection of dentists or dental staff acquired at the practice.

Regarding patient infection, 3.6\% had patients infected 1-2 weeks after visiting the practice. It is important to reiterate that the infection's true origin cannot be confirmed, as transmission could have taken place outside the dental practices or anywhere else. Overall, 13.9\% treated COVID-19 positive patients.

South Africa presented a higher infection rate and more frequent treatment of COVID-19 patients than all other countries combined. This is the first study to provide information on COVID-19 infections among the South African dental community to the best of our knowledge.

The occupational COVID-19 risk of dentists and dental staff has been estimated to be high due to the infective potential of saliva droplets and dental-generated aerosols. ${ }^{10}$ There is limited data on COVID-19 infection rates for dentists and dental staff. A study from the ADA estimated the infection rate for US dentists at $0.9 \%$, with $3.7 \%$ of survey respondents having tested positive via respiratory samples. ${ }^{8}$ Brazil's National Dentistry Council estimated dentists' infection rate to be $0.2 \%$ based on the country's total number of infections. ${ }^{9}$ In the current survey, infection of any member of the dental team was reported by participants, with no separate data on dentists. The higher rate observed in the present study can be the result of selection bias. Dentists who did not have infections in their practices might have been less likely to participate, which could lead to overestimation of COVID-19 infection rates.

The prevalence rate of COVID-19 in the general population has been estimated to be around $2 \% .{ }^{11}$ In $44 \%$ of frontline healthcare workers in the UK, there was evidence of SARS-CoV-2 infection. ${ }^{12}$ In a meta-analysis, the prevalence of infection among healthcare workers worldwide ranged from $0.4 \%$ to $57.1 \%$, with a pooled estimate of $11 \% .{ }^{13}$ Thus, the reported infection rate in the current survey was higher than the infection rate of US and Brazilian dentists, but was in accordance with infection rates for healthcare professionals.

Considering the general population infection rate of $2 \%$ for SARS-CoV-211, findings from this survey suggest that visiting a dental practice could increase the risk for infection, as $4.3 \%$ of participants reported patient infection after a visit to the dental practice. But these results should be interpreted with caution, since tracing the origin of COVID-19 infections is challenging and multiple risk factors have been implicated in the epidemiology of the coronavirus disease, including host defense, underlying health conditions, adherence to social distancing, exposure to infected individuals, age, and personal hygiene habits. ${ }^{14}$

Findings from this survey indicate that nearly $14 \%$ of dental practices had COVID-19 positive patients who needed dental treatment, which increases the occupational COVID-19 risk of the dentist and dental staff and highlights the need for strict infection control measures to protect the dental team. In a survey from Brazil, 5.3\% of dentists reported treatment of COVID-19 patients. ${ }^{15}$

Although an explanation for the higher treatment of COVID -19 patients in South Africa cannot be readily found, the higher number of dentist per inhabitants in Brazil is twice as high as recommended by the WHO $(1: 753),{ }^{16}$ which is extraordinarily higher than the dentist to population ratio of $1: 8,900$ in South Africa. ${ }^{17}$

In this survey, only $1.1 \%$ of respondents attributed COVID -19 infections of dental practitioners and their staff to dental practices, which is low compared to the frequency of treatment of COVID-19 positive patients (13.9\%). In addition, treatment of confirmed COVID-19 positive patients did not increase the reported infection rates for dentists and their staff, indicating that infection control protocols are being successfully followed when COVID-19 patients require emergency treatment. 
The biggest challenge in dental settings is likely the asymptomatic carriers of the coronavirus. Identification of infected and asymptomatic patients is essential to limit the infection risk of dental practitioners and staff. For this reason, accurate and affordable point-of-care diagnostic tests for SARS-CoV-2 that provide results within minutes, are warranted. This can facilitate the decisionmaking process related to clinical patient care, thus reducing the pressure on health professionals. ${ }^{18}$ However, a recent Cochrane meta-analysis reported that most commercialized point-of-care tests still lack data on accuracy and usefulness in clinical practice. ${ }^{19}$

The infection rate among dental professionals can be affected by multiple factors that were not evaluated in the survey, such as using PPE and other infection control protocols, access to diagnostic tests, and the number of patients who visited the dental practice during the pandemic, among others. Results from this survey rely on the accuracy of COVID-19 diagnostic tests, which can be subject to false-negative and false-positive results, as highlighted in the survey study performed by the ADA. Lastly, even though respondents reported that most infections of dentists and dental staff were communityacquired, the actual source of COVID-19 infection is difficult to ascertain, as mentioned previously, making these findings difficult to interpret. ${ }^{20}$

Because of the infectious and inflammatory nature of ubiquitous dental diseases such as caries and periodontal disease, ${ }^{21}$ not all treatments can be delayed as it can be detrimental for oral and general health. Also, likely, most COVID-19 patients who develop acute problems cannot wait until the infection is over to receive emergency care. Therefore, optimization of infection control protocols is mandatory in dental settings to protect dental professionals, staff, and patients in pandemics. ${ }^{22}$ This is of particular importance given the increased infectivity and higher transmissibility of emerging SARS-Cov-2 new variants. ${ }^{23}$

\section{CONCLUSIONS}

The work environment of dental professionals leads to high exposure to respiratory diseases such as coronavirus disease. The COVID-19 infection rate in dental practices was higher than the general population's estimated rate but comparable to most reported rates for healthcare workers.

South Africa had more infections and more frequent treatment of COVID-19 patients. These results support the need for strict infection control measures in dental practices to decrease the risk of transmission of SARS -CoV-2 to the dental team and patients during oral care delivery.

\section{References}

1. Jamal M, Shah M, Almarzooqi SH, et al. Overview of transnational recommendations for COVID-19 transmission control in dental care settings. Oral Dis. 2020;00;1-10. doi: doi. org/10.1111/odi.13431.

2. Peng X, Xu X, Li Y, Cheng L, Zhou X, Ren B. Transmission routes of 2019-nCoV and controls in dental practice. International Journal of Oral Science. 2020; 12: 1-6. doi: doi.org/10.1038/ s41368-020-0075-9.

3. World Health Organization. Considerations for the provision of essential oral health services: Interim Guidance 2020, 3 August 2020. World Health Organization. https://apps.who.int/iris/ handle/10665/333625. License: CC BY-NC-SA 3.0 IGO.

4. UK Office for National Statistics. Which occupations have the highest potential exposure to the coronavirus (COVID-19)? 2020. Available from: https://www.ons.gov.uk/employmentandlabour market/peopleinwork/employmentandemployeetypes/articles/ whichoccupationshavethehighestpotentialexposuretothecoronaviruscovid19/2020-05-11.

5. Brian Z, Weintraub JA. Oral Health and COVID-19: Increasing the need for prevention and access. Prev Chronic Dis. 1 Aug 2020; 17: 200266. doi: 10.5888/pcd17.200266.

6. Centers for Disease Control and Prevention Guidance for Dental Settings. Interim Infection Prevention and Control Guidance for Dental Settings During the COVID-19 Response. 4 Dec 2020. Available from: https://www.cdc.gov/coronavirus/2019-ncov/ hcp/dental-settings.html

7. American Dental Association. ADA COVID-19 Center for Dentists 2020. Available from: https://success.ada.org/en/practicemanagement/patientsinfectious-diseases-2019-novel-coronavirus.

8. Estrich CG, Mikkelsen M, Morrissey R, et al. Estimating COVID -19 prevalence and infection control practices among US dentists. J Am Dent Assoc. 2020;151(11):815-24. doi: 10.1016/j. adaj.2020.09.005.

9. Conselho Federal de Odontologia. Relatório - COVID-19 Odontologia. 2020. Available from: https://website.cfo.org.br/ wp-content/uploads/2020/07/Relatório-covid19-OdontologiaCirurgioes-Dentistas-1.pdf.

10. Li Y, Ren B, Peng X, et al. Saliva is a non-negligible factor in the spread of COVID-19. Molecular Oral Microbiology. 2020; 35: 141-5. doi: 10.1111/omi.12289.

11. Toulis P. Estimation of Covid-19 Prevalence from Serology Tests: A Partial Identification Approach. J Econom. 2021; 220(1): 193-213. doi: 10.1016/j.jeconom.2020.10.005.

12. Houlihan CF, Vora N, Byrne T, et al. Pandemic peak SARS -CoV-2 infection and seroconversion rates in London frontline health-care workers. The Lancet. 2020; 396: e6-7. doi: 10.1016 /S0140-6736(20)31484-7.

13. Gómez-Ochoa S, Franco O, Rojas LZ, et al. COVID-19 in health-care workers: a living systematic review and metaanalysis of prevalence, risk factors, clinical characteristics, and outcomes. Am J Epidemiol. 2020; 190(1): 161-75. doi: 10.1093 /aje/kwaa191 doi: 10.1111/idj.12332.

14. Lakshmi Priyadarsini S, Suresh M. Factors influencing the epidemiological characteristics of pandemic COVID 19: A TISM approach. Int J Healthc Manag. 2020; 13(2): 89-98. doi: doi. org/10.1080/20479700.2020.1755804.

15. Moraes RR, Correa MB, Queiroz AB, et al. COVID-19 challenges to dentistry in the new pandemic epicenter: Brazil. PLoS One. 2020; 15(11): e0242251. doi: 10.1371/journal. pone.0242251.

16. Casado PL, Quinelato V, Cataldo P, et al. Dental genetics in Brazil: Where we are. Molecular Genetics and Genomic Medicine. 2018; 6: 689-701. doi: 10.1002/mgg3.457.

17. Bhayat A, Chikte U. The changing demographic profile of dentists and dental specialists in South Africa: 2002-2015. Int Dent J. 2018; 68(2): 91-6. 
18. Parupudi T, Panchagnula N, Muthukumar S, Prasad S. Evidence-based point-of-care technology development during the COVID-19 pandemic. Biotechniques. 2021; 70(1): 58-67. doi: 10.2144/btn-2020-0096.

19. Dinnes J, Deeks JJ, Adriano A, et al. Rapid, point-of-care antigen and molecular-based tests for diagnosis of SARSCoV-2 infection. Cochrane Database Syst Rev. 2020; 8: CD0 13705. doi: 10.1002/14651858.CD013705.

20. Mbunge $\mathrm{E}$. Integrating emerging technologies into COVID-19 contact tracing: Opportunities, challenges and pitfalls. Diabetes Metab Syndr Clin Res Rev. 2020; 14(6): 1631-6. doi: 10.1016/j. dsx.2020.08.029.

21. Dye BA. The Global Burden of Oral Disease: Research and Public Health Significance. J Dent Res. 2017; 96(4): 361-3. doi: 10.1177/0022034517693567.
22. Banakar M, Bagheri Lakarani K, Jafarpour D, Moayedi S, Banakar MH, Mohammadsadeghi A. COVID-19 transmission risk and protective protocols in dentistry: A systematic review. BMC Oral Health. 2020 Oct 8; 20(1): 275. doi: 10.1186/ s12903-020-01270-9.

23. Korber B, Fischer WM, Gnanakaran S, et al. Tracking Changes in SARS-CoV-2 Spike: Evidence that D614G Increases Infectivity of the COVID-19 Virus. Cell. 20 Aug 2020; 182(4): 812-27. e19. doi: 10.1016/j.cell.2020.06.043.

\section{Do the CPD questionnaire on page 435}

The Continuous Professional Development (CPD) section provides for twenty general questions and five ethics questions. The section provides members with a valuable source of CPD points whilst also achieving the objective of CPD, to assure continuing education. The importance of continuing professional development should not be underestimated, it is a career-long obligation for practicing professionals.

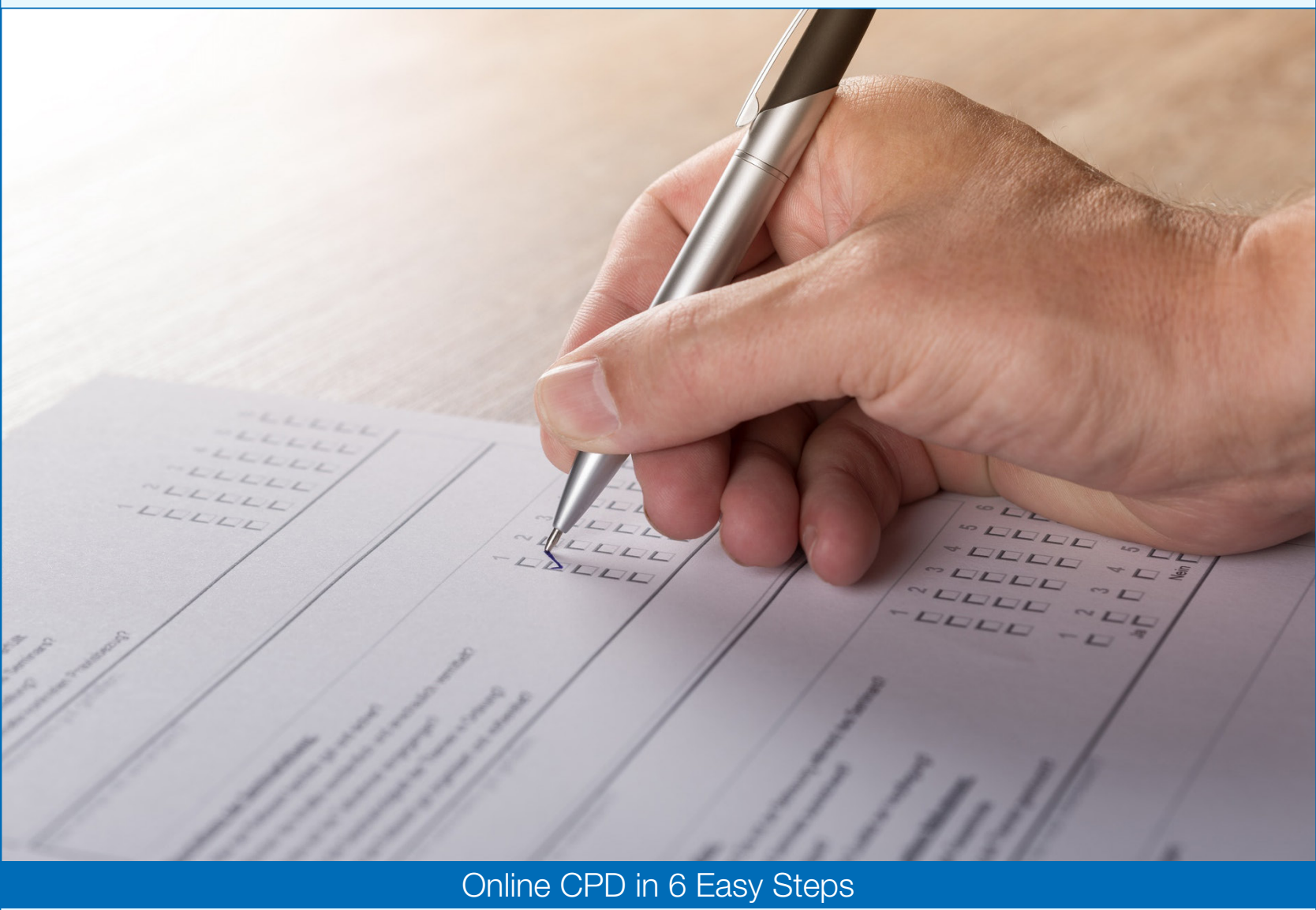

1 Go to the SADA website www.sada.co.za.

2 Log into the 'member only' section with your unique SADA username and password.

3 Select the CPD navigation tab.

4 Select the questionnaire that you wish to complete.

5 Enter your multiple choice answers. Please note that you have two attempts to obtain at least $70 \%$.

6 View and print your CPD certificate. 